\title{
Algorithms and Conditional Lower Bounds for Planning Problems
}

\author{
Krishnendu Chatterjee \\ IST Austria \\ krish.chat@ist.ac.at
}

\author{
Wolfgang Dvořák \\ TU Wien \\ dvorak@dbai.tuwien.ac.at
}

\author{
Monika Henzinger, Alexander Svozil \\ University of Vienna \\ monika.henzinger@univie.ac.at, alexander.svozil@univie.ac.at
}

\begin{abstract}
We consider planning problems for graphs, Markov decision processes (MDPs), and games on graphs. While graphs represent the most basic planning model, MDPs represent interaction with nature and games on graphs represent interaction with an adversarial environment. We consider two planning problems where there are $k$ different target sets, and the problems are as follows: (a) the coverage problem asks whether there is a plan for each individual target set; and (b) the sequential target reachability problem asks whether the targets can be reached in sequence. For the coverage problem, we present a linear-time algorithm for graphs, and quadratic conditional lower bound for MDPs and games on graphs. For the sequential target problem, we present a linear-time algorithm for graphs, a sub-quadratic algorithm for MDPs, and a quadratic conditional lower bound for games on graphs. Our results with conditional lower bounds establish (i) modelseparation results showing that for the coverage problem MDPs and games on graphs are harder than graphs and for the sequential reachability problem games on graphs are harder than MDPs and graphs; and (ii) objective-separation results showing that for MDPs the coverage problem is harder than the sequential target problem.
\end{abstract}

\section{Introduction}

Planning models. One of the basic and fundamental algorithmic problems in artificial intelligence is the planning problem (LaValle 2006; Russell and Norvig 2010). The classical models in planning are as follows:

- Graphs. The most basic planning problems are graph search problems (LaValle 2006; Russell and Norvig 2010).

- MDPs. In the presence of interaction with nature, the graph model is extended with probabilities or stochastic transitions, which gives rise to Markov decision processes (MDPs) (Howard 1960; Puterman 1994; Filar and Vrieze 1997; Papadimitriou and Tsitsiklis 1987).

- Games on graphs. In the presence of interaction with an adversarial environment, the graph model is extended to AND-OR graphs (or games on graphs) (Mahanti and Bagchi 1985; Hansen and Zilberstein 1998).

Copyright (C) 2018, Association for the Advancement of Artificial Intelligence (www.aaai.org). All rights reserved.
Thus graphs, MDPs, and games on graphs are the fundamental models for planning.

Planning objectives. The planning objective represents the goal that the planner seeks to achieve. Some basic planning objectives are as follows:

- Basic target reachability. Given a set $T$ of target vertices the planning objective is to reach some target vertex from the starting position.

- Coverage Objective. In case of coverage there are $k$ different target sets, namely, $T_{1}, T_{2}, \ldots, T_{k}$, and the planning objective asks whether for each $1 \leq i \leq k$ the basic target reachability with target set $T_{i}$ can be achieved. The coverage models the following scenarios: Consider that there is a robot or a patroller, and there are $k$ different target locations, and if an event or an attack happens in one of the target locations, then that location must be reached. However, the location of the event or the attack is not known in advance and the planner must be prepared that the target set could be any of the $k$ target sets.

- Sequential target reachability. In case of sequential targets there are $k$ different target sets, namely, $T_{1}, T_{2}, \ldots, T_{k}$, and the planning objective asks first to reach $T_{1}$, then $T_{2}$, then $T_{3}$ and so on. This represents the scenario that there is a sequence of tasks that the planner must achieve.

The above are the most natural planning objectives and have been studied in the literature, e.g., in robot planning (KressGazit, Fainekos, and Pappas 2009; Kaelbling, Littman, and Cassandra 1998; Choset 2005).

Planning questions. For the above planning objectives the basic planning questions are as follows: (a) for graphs, the question is whether there exists a plan (or a path) such that the planning objective is satisfied; (b) for MDPs, the basic question is whether there exists a policy such that the planning objective is satisfied almost-surely (i.e., with probability 1); and (c) for games on graphs, the basic question is whether there exists a policy that achieves the objective irrespective of the choices of the adversary. The almost-sure satisfaction for MDPs is also known as the strong cyclic planning in the planning literature (Cimatti et al. 2003), and games on graphs question represent planning in the presence of a worst-case adversary (Mahanti 


\begin{tabular}{|c|c|c|c|}
\hline Obj/Model & Graphs & MDPs & Games on graphs \\
\hline Basic target & $O(m)$ & $O\left(m \cdot n^{2 / 3}\right)$ & $O(m)$ \\
\hline Coverage objective & $O\left(m+\sum_{i=1}^{k}\left|T_{i}\right|\right)$ & $\begin{array}{l}O\left(m \cdot n^{2 / 3}+k \cdot m\right) \\
\tilde{\boldsymbol{\Omega}}(\boldsymbol{k} \cdot \boldsymbol{m})[\mathrm{Thm} .1,2]\end{array}$ & $\begin{array}{c}O(k \cdot m) \\
\tilde{\boldsymbol{\Omega}}(\boldsymbol{k} \cdot \boldsymbol{m})[\text { Thm. 3,4] }\end{array}$ \\
\hline Sequential target & $\begin{array}{c}O\left(m+\sum_{i=1}^{k}\left|T_{i}\right|\right) \\
\text { [Thm. 6] }\end{array}$ & $\begin{array}{c}O\left(m \cdot n^{2 / 3}+\sum_{i=1}^{k}\left|T_{i}\right|\right) \\
{[\text { Thm. 5] }}\end{array}$ & $\begin{array}{c}O(k \cdot m) \\
\tilde{\boldsymbol{\Omega}}(\boldsymbol{k} \cdot \boldsymbol{m})[\mathrm{Thm} .7,8]\end{array}$ \\
\hline
\end{tabular}

Table 1: Algorithmic bounds where $n$ and $m$ are the number of vertices and edges of the underlying model, and $k$ denotes the number of different target sets. The $\tilde{\Omega}(\cdot)$ bounds are conditional lower bounds (CLBs) under the BMM conjecture and SETH. They establish that polynomial improvements over the given bound are not possible, however, polylogarithmic improvements are not excluded. Note that CLBs are quadratic for $k=\Theta(n)$. The new results are highlighted in boldface.

and Bagchi 1985; Hansen and Zilberstein 1998) (aka adversarial planning, strong planning (Maliah et al. 2014), or conformant/contingent planning (Bonet and Geffner 2000; Hoffmann and Brafman 2005; Palacios and Geffner 2007)).

Algorithmic study. In this work, we consider the algorithmic study of the planning questions for the natural planning objectives for graphs, MDPs, and games on graphs. For all the above questions, polynomial-time algorithms exist. When polynomial-time algorithms exist, proving an unconditional lower bound is extremely rare. A new approach in complexity theory aims to establish conditional lower bound (CLB) results based on some well-known conjecture. Two standard conjectures for CLBs are as follows: The (a) Boolean matrix multiplication (BMM) conjecture which states that there is no sub-cubic combinatorial algorithm for boolean matrix multiplication; and the (b) Strong exponential-time hypothesis (SETH) which states that there is no sub-exponential time algorithm for the SAT problem. Many CLBs have been established based on the above conjectures, e.g., for dynamic graph algorithms, string matching (Abboud and Williams 2014; Bringmann and Künnemann 2015).

Previous results and our contributions. We denote by $n$ and $m$ the number of vertices and edges of the underlying model, and $k$ denotes the number of different target sets. For the basic target reachability problem, while the graphs and games on graphs problem can be solved in linear time (Beeri 1980; Immerman 1981), the current best-known bound for MDPs is $O\left(m \cdot n^{2 / 3}\right)$ (Chatterjee and Henzinger 2014; Chatterjee et al. 2016). For the coverage and sequential target reachability, an $O(k \cdot m)$ upper bound follows for graphs and games on graphs, and an $O\left(m \cdot n^{2 / 3}+k \cdot m\right)$ upper bound follows for MDPs. Our contributions are as follows:

1. Coverage problem: First, we present an $O(m+$ $\left.\sum_{i=1}^{k}\left|T_{i}\right|\right)$ time algorithm for graphs; second, we present an $\Omega(k \cdot m)$ lower bound for MDPs and games on graphs, both under the BMM conjecture and the SETH. Note that for graphs our upper bound is linear time, however, if each $\left|T_{i}\right|$ is constant and $k=\theta(n)$, for MDPs and games on graphs the CLB is quadratic.

2. Sequential target problem: First, we present an $O(m+$ $\left.\sum_{i=1}^{k}\left|T_{i}\right|\right)$ time algorithm for graphs; second, we present an $O\left(m \cdot n^{2 / 3}+\sum_{i=1}^{k}\left|T_{i}\right|\right)$ time algorithm for MDPs; and third, we present an $\Omega(k \cdot m)$ lower bound for games on graphs, both under the BMM conjecture and the SETH. The summary of the results is presented in Table 1. Our most interesting results are the conditional lower bounds for MDPs and game graphs for the coverage problem, the subquadratic algorithm for MDPs with sequential targets, and the conditional lower bound for game graphs with sequential targets.

Practical Significance. The sequential reachability and coverage problems we consider are the tasks defined in (KressGazit, Fainekos, and Pappas 2009, Section II. PROBLEM FORMULATION, 3) System Specification), where the problems have been studied for games on graphs (Section IV. DISCRETE SYNTHESIS) and mentioned as future work for MDPs (Section I. INTRODUCTION, A. Related Work). The applications of these problems have been demonstrated in robotics applications. We present a complete algorithmic picture for games on graphs and MDPs, settling open questions related to games and future work mentioned in (KressGazit, Fainekos, and Pappas 2009).

Theoretical Significance. Our results present a very interesting algorithmic picture for the natural planning questions in the fundamental models.

1. First, we establish results showing that some models are harder than others. More precisely,

- for the basic target problem, the MDP model seems harder than graphs/games on graphs (linear-time algorithm for graphs and games on graphs, and no such algorithms are known for MDPs);

- for the coverage problem, MDPs, and games on graphs are harder than graphs (linear-time algorithm for graphs and quadratic CLBs for MDPs and games on graphs);

- for the sequential target problem, games on graphs are harder than MDPs and graphs (linear-time upper bound for graphs and sub-quadratic upper bound for MDPs, whereas quadratic CLB for games on graphs).

In summary, we establish model-separation results with CLBs: For the coverage problem, MDPs and games on graphs are algorithmically harder than graphs; and for the sequential target problem, games on graphs are algorithmically harder than MDPs and graphs.

2. Second, we also establish objective-separation results. For the model of MDPs consider the different objectives: both for basic target and sequential target reachability the upper bound is sub-quadratic and in contrast to the coverage problem, we establish a quadratic CLB. 
Related works on other models. In this work, our focus lies on the algorithmic complexity of fundamental planning problems and we consider explicit state-space graphs, MDPs, and games, where the complexities are polynomial. The explicit model and algorithms for it are widely considered: (LaValle 2006)[Chapter 2.1 Discrete Feasible Planning], (Kress-Gazit, Fainekos, and Pappas 2009)[Section IV. DISCRETE SYNTHESIS] and (Chatterjee and Henzinger 2014)[Section 2.1. Definitions. Alternating game graphs.]. In other representations such as the factored model, the complexities are higher (NP-complete), and then heuristics are the focus (e.g., (Hansen and Zilberstein 1998)) rather than the algorithmic complexity. Notable exceptions are the work on parameterized complexity of planning problems (see, e.g., (Kronegger, Pfandler, and Pichler 2013)) and Conditional Lower Bounds showing that certain planning problems do not admit subexponential time algorithms (Aghighi et al. 2016; Bäckström and Jonsson 2017).

\section{Preliminaries}

Markov Decision Processes (MDPs). A Markov decision process $(M D P) P=\left((V, E),\left\langle V_{1}, V_{R}\right\rangle, \delta\right)$ consists of a finite set of vertices $V$ partitioned into the player-1 vertices $V_{1}$ and the random vertices $V_{R}$, a finite set of edges $E \subseteq(V \times V)$, and a probabilistic transition function $\delta$. The probabilistic transition function maps every random vertex in $V_{R}$ to an element of $\mathcal{D}(V)$, where $\mathcal{D}(V)$ is the set of probability distributions over the set of vertices $V$. A random vertex $v$ has an edge to a vertex $w \in V$, i.e. $(v, w) \in E$ iff $\delta(v)[w]>0$.

Game Graphs. A game graph $\Gamma=\left((V, E),\left\langle V_{1}, V_{2}\right\rangle\right)$ consists of a finite set of vertices $V$, a finite set of edges $E$ and a partition of the vertices $V$ into player-1 vertices $V_{1}$ and the adversarial player-2 vertices $V_{2}$.

Graphs. Graphs are a special case of MDPs with $V_{R}=\emptyset$ as well as special case of game graphs with $V_{2}=\emptyset$.

Remark 1. Note that a standard way to define MDPs is to consider finite vertices with actions, and the probabilistic transition function is defined for every vertex and action. In our model, the choice of actions is represented as the choice of edges at player-1 vertices and the probabilistic transition function is represented by the random vertices. This allows us to treat MDPs and game graphs in a uniform way, and graphs can be described easily as a special case of MDPs.

Plays. A play is an infinite sequence $\omega=\left\langle v_{0}, v_{1}, v_{2}, \ldots\right\rangle$ of vertices such that each $\left(v_{i-1}, v_{i}\right) \in E$ for all $i \geq 1$. The set of all plays is denoted with $\Omega$. A play is initialized by placing a token on an initial vertex. If the token is on a vertex owned by a player (such as player 1 in MDPs, or player 1 or player 2 in game graphs), then the respective player moves the token along one of the outgoing edges, whereas if the token is at a random vertex $v \in V_{R}$, then the next vertex is chosen according to the probability distribution $\delta(v)$. Thus an infinite sequence of vertices (or an infinite walk) is formed which is a play.

Policies. Policies are recipes for players to extend finite prefixes of plays. Formally, a player-i policy is a function $\sigma_{i}: V^{*} \cdot V_{i} \mapsto V$ which maps every finite prefix $\omega \in V^{*} \cdot V_{i}$ of a play that ends in a player-i vertex $v$ to a successor vertex $\sigma_{i}(\omega) \in V$, i.e., $\left(v, \sigma_{i}(\omega)\right) \in E$. A player-1 policy is memoryless or stationary if $\sigma_{i}(\omega)=\sigma_{i}\left(\omega^{\prime}\right)$ for all $\omega, \omega^{\prime} \in V^{*} \cdot V_{1}$ that end in the same vertex $v \in V_{1}$, i.e., the policy does not depend on the entire prefix, but only on the last vertex.

Outcome of policies. Outcome of policies are as follows:

- In graphs, given a starting vertex, a policy for player 1 induces a unique play in the graph.

- In game graphs, given a starting vertex $v$, and policies $\sigma_{1}, \sigma_{2}$ for player 1 and player 2 respectively, the outcome is a unique play $\omega\left(v, \sigma_{1}, \sigma_{2}\right)=\left\langle v_{0}, v_{1}, v_{2}, \ldots\right\rangle$, where $v_{0}=v$ and for all $i \geq 0$ if $v_{i} \in V_{1}$ then $\sigma_{1}\left(\left\langle v_{0}, \ldots, v_{i}\right\rangle\right)=v_{i+1}$ and if $v_{i} \in V_{2}$, then $\sigma_{2}\left(\left\langle v_{0}, \ldots, v_{i}\right\rangle\right)=v_{i+1}$.

- In MDPs, given a starting vertex $v$ and a policy $\sigma$ for player 1 , there is a unique probability measure over $\Omega$ which is denoted as $\operatorname{Pr}_{v}^{\sigma}(\cdot)$.

Objectives and winning. In general, an objective $\phi$ is a measurable subset of $\Omega$. A play $\omega \in \Omega$ achieves the objective if $\omega \in \phi$. We consider the following notion of winning:

- Almost-sure winning. In MDPs, a player-1 policy $\sigma$ is almost-sure (a.s.) winning from a starting vertex $v \in V$ for an objective $\phi$ iff $\operatorname{Pr}_{v}^{\sigma}(\phi)=1$.

- Winning. In game graphs a policy $\sigma_{1}$ is winning for player 1 from a starting vertex $v$ iff the resulting play achieves the objective irrespective of the policy of player 2, i.e., for all $\sigma_{2}$ we have $\omega\left(v, \sigma_{1}, \sigma_{2}\right) \in \phi$.

Note that in the special case of graphs both of the above winning notions requires that there exists a play from $v$ that achieves the objective.

Remark 2. In MDPs we consider a.s. winning planning for which the precise transition probabilities of the transition function $\delta$ does not matter, but only the support of the transition function is relevant. The a.s. winning notion we use corresponds to the strong cyclic planning problem. Intuitively, if we visit a random vertex in an MDPs infinitely often then all its successors are visited infinitely often. This represents the local fairness condition (Clarke, Grumberg, and Peled 1999). Therefore, when we consider the MDP question only the underlying graph structure along with the partition is relevant, and the transition function $\delta$ can be treated as a uniform distribution over the support.

We have defined the notion of objectives in general above, and below we consider specific objectives that are natural in planning problems. They are all variants of one of the most fundamental objectives in computer science, namely, reachability objectives.

Basic Target Reachability. For a set $T \subseteq V$ of target set vertices, the basic target reachability objective is the set of infinite paths that contain a vertex of $T$, i.e., $\operatorname{Reach}(T)=$ $\left\{\left\langle v_{0}, v_{1}, v_{2}, \ldots\right\rangle \in \Omega \mid \exists j \geq 0: v_{j} \in T\right\}$.

Coverage Objective. For $k$ different target sets, namely $T_{1}, T_{2}, \ldots, T_{k}$, the coverage objective asks whether for each $1 \leq i \leq k$ the basic target reachability objective $\operatorname{Reach}\left(T_{i}\right)$ can be achieved. More precisely, given a starting vertex $v$, one asks whether for every $1 \leq i \leq k$ there is a policy $\sigma_{1}^{i}$ to ensure winning (resp., a.s. winning) for the objective $\operatorname{Reach}\left(T_{i}\right)$ from $v$ for game graphs (resp., MDPs). 


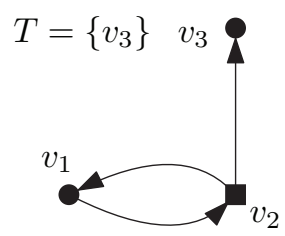

Figure 1: Example illustrating the difference between MDPs and Game Graphs for the reachability objective Reach $(T)$.

Sequential Target Reachability. For a tuple of vertex sets $\mathcal{T}=\left(T_{1}, T_{2}, \ldots, T_{k}\right)$ the sequential target reachability objective is the set of infinite paths that contain a vertex of $T_{1}$ followed by a vertex of $T_{2}$ and so on up to a vertex of $T_{k}$, i.e., $\operatorname{Seq}(\mathcal{T})=\left\{\left\langle v_{0}, v_{1}, v_{2}, \ldots\right\rangle \in \Omega \mid \exists j_{1}, j_{2}, \ldots j_{k}: v_{j_{1}} \in\right.$ $T_{1}, v_{j_{2}} \in T_{2}, \ldots, v_{j_{k}} \in T_{k}$ and $\left.j_{1} \leq j_{2} \leq \cdots \leq j_{k}\right\}$.

Example: Difference between MDPs and Game Graphs. Let the graph $G=(V, E)$ be defined as follows: Let $V=\left\{v_{1}, v_{2}, v_{3}\right\}$ and $E=\left\{\left(v_{1}, v_{2}\right),\left(v_{2}, v_{1}\right),\left(v_{2}, v_{3}\right)\right\}$. Let $T=\left\{v_{3}\right\}$ be a target set. We will now consider Reach $(T)$ for the MDP $P=\left(G,\left\langle V_{1}, V_{R}\right\rangle, \delta\right)$ and the game graph $\Gamma=\left(G,\left\langle V_{1}, V_{2}\right\rangle\right)$. Let $V_{1}=\left\{v_{1}, v_{3}\right\}$ and $V_{2}=V_{R}=\left\{v_{2}\right\}$. The example is illustrated in Figure 1. The adversary always chooses to go to $v_{1}$ and the target is never reached from $v_{1}$. On the other hand, if $v_{2}$ is probabilistic whenever the token is at $v_{2}$ it is moved to $v_{3}$ with non-zero probability. That is, almost-surely the transition from $v_{2}$ to $v_{3}$ is taken eventually, i.e. $v_{3}$ is reached almost-surely. Thus, reachability in MDPs does not imply reachability in game graphs.

Relevant parameters. We will consider the following parameters: $n$ denotes the number of vertices, $m$ denotes the number of edges and $k$ will either denote the number of target sets in the coverage problem or the size of the tuple of target sets in the sequential target reachability problem.

Algorithmic study. In this work we study the above basic planning objectives for graphs, game graphs (i.e., winning in game graphs), and MDPs (a.s. winning in MDPs). Our goal is to clarify the algorithmic complexity of the above questions with improved algorithms and conditional lower bounds. We define the conjectured lower bounds for conditional lower bounds below.

\section{Conjectured Lower Bounds}

Results from classical complexity are based on standard complexity-theoretical assumptions, e.g., $\mathbf{P} \neq$ NP. Similarly, we derive polynomial lower bounds which are based on widely believed, conjectured lower bounds on well studied algorithmic problems. In this work the lower bounds we derive depend on the popular conjectures below:

First of all, we consider conjectures on Boolean Matrix Multiplication (Williams and Williams 2018)[Theorem 6.1] and triangle detection in graphs (Abboud and Williams 2014)[Conjecture 2], which are the basis for lower bounds on dense graphs. A triangle in a graph is a triple $x, y, z$ of vertices such that $(x, y),(y, z),(z, x) \in E$. We will for the rest of this work assume that vertices contain at least one outgoing edge and no self-loops in instances of Triangle.
This can be easily established by linear time preprocessing. See Remark 3 for a discussion of the term "combinatorial algorithm",

Conjecture 1 (Combinatorial Boolean Matrix Multiplication Conjecture (BMM)). There is no $O\left(n^{3-\epsilon}\right)$ time combinatorial algorithm for computing the boolean product of two $n \times n$ matrices for any $\epsilon>0$.

Conjecture 2 (Strong Triangle Conjecture (STC)). There is no $O\left(\min \left\{n^{\omega-\epsilon}, m^{2 \omega /(\omega+1)-\epsilon}\right\}\right)$ expected time algorithm and no $O\left(n^{3-\epsilon}\right)$ time combinatorial algorithm that can detect whether a graph contains a triangle for any $\epsilon>0$, where $\omega<2.373$ is the matrix multiplication exponent.

Williams and Williams (2018, Theorem 6.1) showed that $\mathrm{BMM}$ is equivalent to the combinatorial part of STC. Moreover, if we do not restrict ourselves to combinatorial algorithms, STC, still gives a super-linear lower bound.

Remark 3 (Combinatorial Algorithms). "Combinatorial" in Conjecture 2 means that it excludes "algebraic methods" (such as fast matrix multiplication (Williams 2012; Le Gall 2014)), which are impractical due to high associated constants. Therefore the term "combinatorial algorithm" comprises only discrete algorithms. Non-combinatorial algorithms usually have the matrix multiplication exponent $\omega$ in the running time. Notice that all algorithms for deciding almost-sure winning conditions in MDPs and winning conditions in games are discrete graph-theoretic algorithms and hence are combinatorial, and thus lower bounds for combinatorial algorithms are of particular interest in our setting. For further discussion consider (Ballard et al. 2012; Henzinger et al. 2015).

Secondly, we consider the Strong Exponential Time Hypothesis (SETH) used also in (Abboud and Williams 2014)[Conjecture 1] introduced by (Impagliazzo and Paturi 1999; Impagliazzo, Paturi, and Zane 1998) for the satisfiability problem of propositional logic and the Orthogonal Vector Conjecture.

The Orthogonal Vectors Problem (OV). Given sets $S_{1}, S_{2}$ of $d$-bit vectors with $\left|S_{1}\right|=\left|S_{2}\right|=N$ and $d=\omega(\log N)$, are there $u \in S_{1}$ and $v \in S_{2}$ such that $\sum_{i=1}^{d} u_{i} \cdot v_{i}=0$ ?

Conjecture 3 (Strong Exponential Time Hypothesis (SETH)). For each $\epsilon>0$ there is a $k$ such that $k$-CNF$S A T$ on $n$ variables and $m$ clauses cannot be solved in $O\left(2^{(1-\epsilon) n}\right.$ poly $\left.(m)\right)$ time.

Conjecture 4 (Orthogonal Vectors Conjecture (OVC))). There is no $O\left(N^{2-\epsilon}\right)$ time algorithm for the Orthogonal Vectors Problem for any $\epsilon>0$.

SETH implies OVC, which is an implication of a result in (Williams 2005) and an explicit reduction is given in the survey article by Vassilevska-Williams (2018, Theorem 3.1). Whenever a problem is provably hard assuming OVC it is thus also hard when assuming SETH. For example, in (Bringmann and Künnemann 2015)[Preliminaries, A. Hardness Assumptions, OVH] the OVC is assumed to prove conditional lower bounds for the longest common subsequence problem. To the best of the author's knowledge, there 
is no connection between the former two and the latter two conjectures.

Remark 4. The conjectures that no polynomial improvements over the best-known running times are possible do not exclude improvements by sub-polynomial factors such as polylogarithmic factors or factors of, e.g., $2^{\sqrt{\log n}}$.

\section{Basic Previous Results}

In this section, we recall the basic algorithmic results about MDPs and game graphs known in the literature that we later use in our algorithms.

Basic result 1: Maximal End-Component Decomposition. Given an MDP $P$, an end-component is a set of vertices $X \subseteq V$ s.t. (1) the subgraph induced by $X$ is strongly connected (i.e., $(X, E \cap X \times X)$ is strongly connected) and (2) all random vertices have their outgoing edges in $X$, i.e., $X$ is closed for random vertices, formally described as: for all $v \in X \cap V_{R}$ and all $(v, u) \in E$ we have $u \in X$. A maximal end-component (MEC) is an end-component which is maximal under set inclusion. The importance of MECs is as follows: (i) first it generalizes strongly connected components (SCCs) in graphs (with $V_{R}=\emptyset$ ) and closed recurrent sets of Markov chains (with $V_{1}=\emptyset$ ); and (ii) in a MEC $X$ from all vertices $u \in X$ every vertex $v \in$ $X$ can be reached almost-surely. The MEC-decomposition of an MDP is the partition of the vertex set into MECs and the set of vertices which do not belong to any MEC. While MEC-decomposition generalizes SCC decomposition of graphs, and SCC decomposition can be computed in linear time (Tarjan 1972, Theorem 13), there is no linear-time algorithm for MEC-decomposition computation. The current best-known algorithmic bound for MEC-decomposition is $O\left(\min \left(n^{2}, m^{1.5}\right)=O\left(m \cdot n^{2 / 3}\right)\right)$ (Chatterjee and Henzinger 2014, Theorem 3.6, Theorem 3.10).

Basic result 2: Reachability in MDPs. Given an MDP $P$ and a target set $T$, the set of starting vertices from which $T$ can be reached almost-surely can be computed in $O(m)$ time given the MEC-decomposition of $P$ (Chatterjee et al. 2016, Theorem 4.1). Moreover, for the basic target reachability problem the current best-known algorithmic bounds are the same as the MEC-decomposition problem, i.e., $O\left(\min \left(n^{2}, m^{1.5}\right)\right)=O\left(m \cdot n^{2 / 3}\right)$ (Chatterjee and Henzinger 2014, Theorem 3.6, Theorem 3.10), and any improvement for the MEC-decomposition algorithm also carries over to the basic target reachability problem.

Basic result 3: Reachability in game graphs. Given a game graph $\Gamma$ and a target set $T$, the set of starting vertices from which player 1 can ensure to reach $T$ against all policies of player 2, is called player- 1 attractor to $T$ and can be computed in $O(m)$ time (Beeri 1980; Immerman 1981).

The above basic results from the literature explain the result of the first row of Table 1 .

\section{Coverage Problem}

In this section we consider the coverage problem. First, we present the algorithms, which are straightforward, and then focus on the conditional lower bounds for MDPs and game graphs, which establish that the existing algorithms cannot be improved under the STC and the OVC.

\section{Algorithms}

We present a linear-time algorithm for graphs and quadratic time algorithm for MDPs and game graphs. The results below present the upper bounds of the second row of Table 1.

Planning in Graphs. For the coverage problem in graphs we are given a graph $G=(V, E)$, a vertex $s \in V$ and target sets $T_{1}, T_{2}, \ldots, T_{k}$. The algorithmic problem is to find out if starting from an initial vertex $v$ the basic target reachability, i.e., Reach $\left(T_{i}\right)$, can be achieved for all $1 \leq i \leq k$. The algorithmic solution is as follows: Compute the BFS tree starting from $s$ and check if all the targets are contained in the resulting BFS tree.

Planning in MDPs and Games. For both MDPs and game graphs with $k$ target sets, the basic algorithm performs $k$ basic reachability computations, i.e., for each target set $T_{i}$, $1 \leq i \leq k$, the basic target reachability for target set $T_{i}$ is computed. (1) For game graphs, using the $O(m)$-time attractor computation (see Basic result 3), we have an $O(k \cdot m)$ time algorithm. (2) For MDPs, the MEC-decomposition followed by $k$ many $O(m)$-time almost-sure reachability computations (see Basic result 2), gives an $O(k \cdot m+$ MEC) time algorithm.

\section{Conditional Lower Bounds}

We present CLBs for the coverage problem in MDPs and game graphs (i.e., the CLBs of the second row of Table 1). For MDPs and game graphs the CLBs complement the quadratic algorithms from the previous subsection. The CLBs are due to reductions from OV and Triangle.

Sparse MDPs. For sparse MDPs we present a conditional lower bound based on OVC. To do that we reduce the OV problem to the coverage problem in MDPs.

Reduction 1. Given two sets $S_{1}, S_{2}$ of d-dimensional vectors, we build the MDP $P$ as follows. The vertices $V$ of the MDP are given by a start vertex $s$, sets of vertices $S_{1}$ and $S_{2}$ representing the sets of vectors and vertices $\mathcal{C}=\left\{c_{i} \mid 1 \leq\right.$ $i \leq d\}$ representing the coordinates of the vectors in the OVC instance. The edges $E$ of $P$ are defined as follows: The start vertex s has an edge to every vertex of $S_{1}$. Furthermore for each $x_{i} \in S_{1}$ there is an edge to $c_{j} \in C$ iff $x_{i}[j]=1$ and for each $y_{i} \in S_{2}$ there is an edge from $c_{j} \in S_{2}$ to $y_{i}$ iff $y_{i}[j]=1$. The set of vertices is partitioned into player-1 vertices $V_{1}=S_{1} \cup \mathcal{C} \cup S_{2}$ and random vertices $V_{R}=\{s\}$.

The reduction is illustrated in Figure 2 (the dashed edges will be used later for the sequential target lower bounds).

Theorem 1. There is no $O\left(m^{2-\epsilon}\right)$ or $O\left((k \cdot m)^{1-\epsilon}\right)$ (for any $\epsilon>0)$ algorithm to check if a vertex $v$ has an a.s. winning policy for the coverage problem in MDPs under Conjecture 4 (i.e., unless OVC and SETH fail).

Proof Sketch. Let $P=\left(V, E,\left\langle V_{1}, V_{R}\right\rangle, \delta\right)$ be the MDP given by Reduction 1 with target sets $T_{i}=\left\{y_{i}\right\}$ for $i=$ $1 \ldots N$. Notice that (a) when starting from $s$ the token is randomly moved to one of the vertices $x_{i}$ and thus player 1 


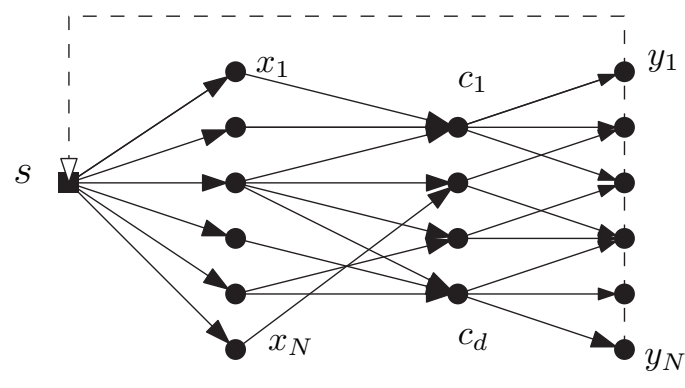

Figure 2: Reduction from OV

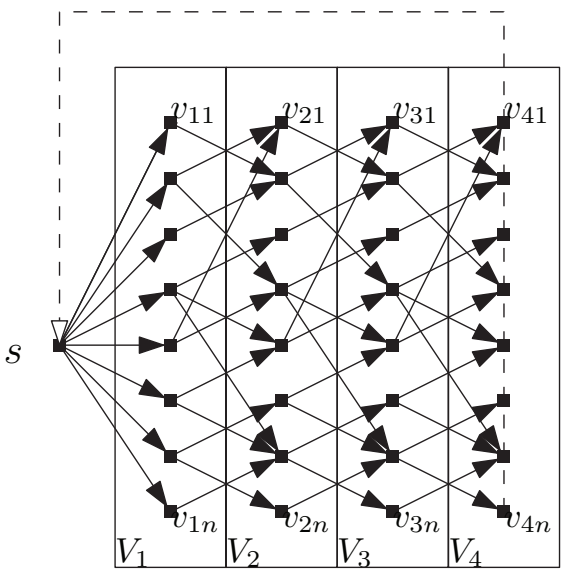

Figure 3: Reduction from Triangle

can reach each $y_{j}$ almost surely from $s$ iff it can reach each $y_{j}$ from each $x_{i}$, and (b) the MDP $P$ is constructed in such a way that there is no path between vertex $x_{i}$ and $y_{j}$ iff the corresponding vectors are orthogonal in the OV instance. That is, player 1 can reach all the target sets a.s. from $s$ iff there are no orthogonal vectors in $S_{1}$ and $S_{2}$. The MDP has only $O(N)$ many vertices and Reduction 1 can be performed in $O(N \log N)$ time (recall that $d=\omega(\log N))$. The number of edges $m$ is $O(N \log N)$ and the number of target sets $k \in \theta(N)$.

Dense MDPs. For dense MDPs we present a conditional lower bound based on boolean matrix multiplication (BMM). Therefore we reduce the Triangle detection problem to the coverage problem in MDPs.

Reduction 2. Given an instance of triangle detection, i.e., a graph $G=(V, E)$, we build the following $M D P P=$ $\left(V^{\prime}, E^{\prime},\left\langle V_{1}^{\prime}, V_{R}^{\prime}\right\rangle, \delta\right)$. The vertices $V^{\prime}$ are given as four copies $V_{1}, V_{2}, V_{3}, V_{4}$ of $V$ and a start vertex s. The edges $E^{\prime}$ of $P$ are defined as follows: There is an edge from $s$ to every $v_{1 i} \in V_{1}$ for $i=1 \ldots n$. In addition for $1 \leq j \leq 3$ there is an edge from $v_{j i}$ to $v_{(j+1) k}$ iff $\left(v_{i}, v_{k}\right) \in E$. The set of vertices $V^{\prime}$ is partitioned into player-1 vertices $V_{1}^{\prime}=\emptyset$ and random vertices $V_{R}^{\prime}=\{s\} \cup V_{1} \cup V_{2} \cup V_{3} \cup V_{4}$.

The reduction is illustrated in Figure 3 (the dashed edges will be used later for the sequential target lower bounds).
Theorem 2. There is no combinatorial $O\left(n^{3-\epsilon}\right)$ or $O((k$. $\left.n^{2}\right)^{1-\epsilon}$ ) algorithm (for any $\epsilon>0$ ) to check if a vertex has an a.s. winning policy for the coverage objective in MDPs under Conjecture 2 (i.e., unless STC and BMM fail). The bounds hold for dense MDPs with $m=\theta\left(n^{2}\right)$.

Proof Sketch. Let $P$ be the MDP given by Reduction 2 with $n$ target sets $T_{1}, \ldots, T_{n}$. The target set $T_{i}=V_{1} \backslash\left\{v_{1 i}\right\} \cup$ $V_{4} \backslash\left\{v_{4 i}\right\}$ for $i=1 \ldots n$. Notice that (a) there is a triangle in the graph $G$ iff there is a path from some vertex $v_{1 i}$ in the first copy of $G$ to the same vertex in the fourth copy of $G, v_{4 i}$, and (b) a path starting in $s$ satisfies the objective, i.e., reaches all target sets a.s., unless it visits a vertex $v_{1 i}$ and also $v_{4 i}$. As each of these paths has non-zero probability player 1 wins almost-surely from $v$ iff there is no such path iff there is no triangle in the original graph. Moreover, the size and the construction time of graph $G^{\prime}$ are linear in the size of the original graph $G$ and we have $k=\theta(n)$ target sets.

Next, we briefly describe how the results for MDPs can be extended to game graphs.

Reduction from $O V$. The random starting vertex in the reduction is changed to a player- 2 vertex. The rest of the reduction stays the same. The proof proceeds as before with the adversary player 2 now overtaking the role of the random choices.

Theorem 3. There is no $O\left(m^{2-\epsilon}\right)$ or $O\left((k \cdot m)^{1-\epsilon}\right.$ algorithm (for any $\epsilon>0$ ) to check if a vertex has a winning policy for the coverage objective with $k$ reachability objectives in game graphs under Conjecture 4 (i.e., unless OVC and SETH fail).

Reduction from Triangle. The random vertices in the reduction are now player- 2 vertices. Notice that the resulting game graph $\Gamma$ has only player- 2 vertices. Now if there is a path starting from $s$ that is not in the defined coverage objective then player 2 would simply choose that one and thus player 1 still wins iff there is no such path, i.e., there is no triangle in the original graph.

Theorem 4. There is no combinatorial $O\left(n^{3-\epsilon}\right)$ or $O((k$. $\left.n^{2}\right)^{1-\epsilon}$ ) algorithm (for any $\epsilon>0$ ) to check whether a vertex $v$ has a winning policy for the coverage objective in game graphs under Conjecture 2 (i.e., unless STC and BMM fail). The bounds hold for dense game graphs with $m=\theta\left(n^{2}\right)$.

\section{Sequential Target Problem}

We consider the sequential target problem in graphs, MDPs and game graphs. In contrast to the quadratic CLB for the coverage problem, quite surprisingly we present a subquadratic algorithm for MDPs, which as a special case gives a linear-time algorithm for graphs. For games, we present a quadratic algorithm and a quadratic CLB.

\section{Algorithms}

The results below present the upper bounds of the third row of Table 1.

Planning in MDPs. We first calculate the MECdecomposition of the MDP. Then each MEC is collapsed 


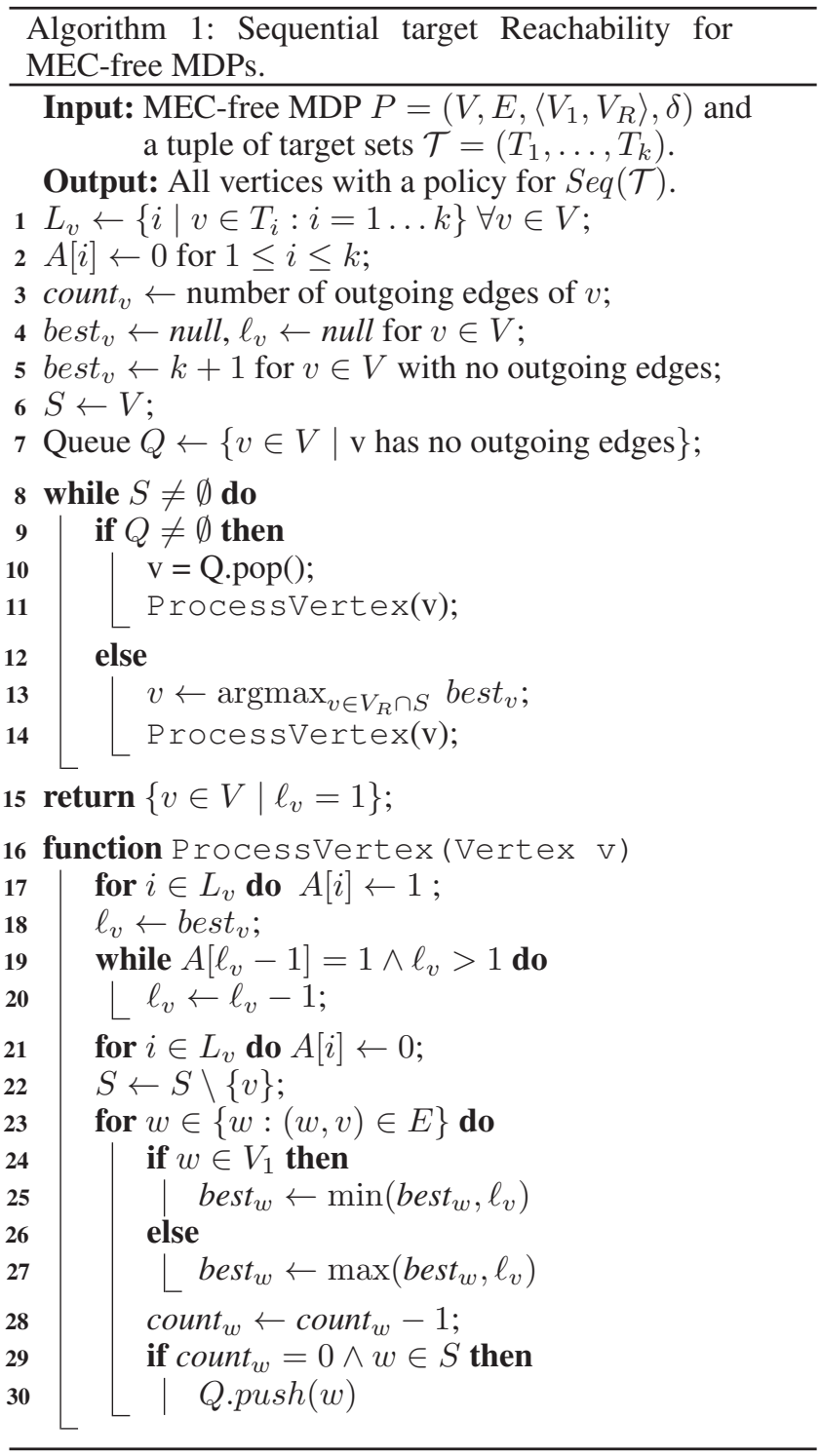

into a single vertex, which we set to be a player-1 vertex. In the target sets, all the vertices of the MEC are replaced by this new vertex. This does not change the reachability conditions of the resulting MDP: Every vertex in a MEC can be visited almost surely from every other vertex in the same MEC, regardless of their type (player-1, random). Thus it suffices to present an algorithm for a MEC-free MDP $P=\left(V, E,\left\langle V_{1}, V_{R}\right\rangle, \delta\right)$ with tuple of target sets $\left(T_{1}, \ldots, T_{k}\right)$. The vertices in $S$ are the vertices that are not processed yet and $S$ is initialized with $V$. Initially, vertices with no outgoing edges are added into a queue $Q$. Throughout the algorithm the queue $Q$ contains the vertices which have not been processed so far but whose successors are already processed. While the queue $Q$ is not empty, a vertex from the queue is processed. When a vertex $v$ is processed the function ProcessVertex $(v)$ is called. The function calculates the label $\ell_{v}$ of the vertex $v$ and updates variables best ${ }_{w}$ and count $_{w}$ of the other vertices. The variable count $_{v}$ counts the number of successors of $v$ that are not yet processed. The label $\ell_{v}$ means vertex $v$ has an almost-sure winning policy for the objective $\operatorname{Seq}\left(\mathcal{T}_{\ell_{v}}\right)$ where $\mathcal{T}_{\ell_{v}}=\left(T_{\ell_{v}}, \ldots, T_{k}\right)$. Note that this means that vertices with label 1 have an almost-sure winning policy for the objective $\operatorname{Seq}(\mathcal{T})$ where $\mathcal{T}=\left\{T_{1}, T_{2}, \ldots T_{k}\right)$. The variables best ${ }_{v}$ are used to store the maximum (for $v \in V_{R}$ ) / minimum (for $v \in V_{1}$ ) label of the already processed successors of $v$. Now when $Q$ is empty, the algorithm has to process a vertex where not all successors have been processed yet. In that case, one considers all the random vertices for which at least one successor has already been processed and chooses the random vertex with maximum best $_{v}$ to process next. (notice that the function argmax ignores arguments with null values). One can show that, as the graph has no MECs, whenever $Q$ is empty (and $S$ is not) there exists such a random vertex. Moreover, whenever $Q$ is empty, all vertices in the set of unprocessed vertices $S$ have a policy that satisfies $S e q\left(\mathcal{T}_{m}\right)$ for $m=\max _{v \in V_{R} \cap S}$ best $_{v}$. Intuitively, this is due to the fact that all vertices $v \in S$ can reach the set of already processed vertices and in the worst case the reached vertex $v^{\prime}$ has $l_{v^{\prime}}=m$ and thus a strategy for $\operatorname{Seq}\left(\mathcal{T}_{m}\right)$. Thus, for the selected vertex $v$, we have that all its successors $w$ will finally have a label $\ell_{w}$ of at most $m$, and, as the current value of best $v_{v}$ is $m$, there is a successor $w$ with $\ell_{w}=m$. Thus, as $v \in V_{R}$, we have that also the final value of best $v$ must be $m$. Hence, one can already process $v$ without knowing the labels of all the successors.

Theorem 5. Given an MDP $P$, a starting vertex $s$ and $a$ tuple of targets $\mathcal{T}=\left(T_{1}, \ldots, T_{k}\right)$, we can calculate whether there is a player-1 policy $\sigma_{1}$ at s for the objective $\operatorname{Seq}(\mathcal{T})$ in $O\left(\operatorname{MEC}+m \log n+\sum_{i=0}^{k}\left|T_{i}\right|\right)$ time.

Running Time. Initializing the algorithm takes $O(m+$ $\left.\sum_{i=0}^{k}\left|T_{i}\right|\right)$ time. This is due to the fact that we calculate $L_{v}$ in $O(n+m+k)$ at Line 1 . The other initialization steps take only $O(m)$ time (Lines 2-6). Now consider the while loop. Every vertex $v \in V$ is processed with a call to ProcessVertex $(\mathrm{v})$ once. Evaluating ProcessVertex $(v)$ takes time linear in the number of incoming edges of $v$ plus $\left|L_{v}\right|$. Summing up over all vertices we obtain a $O\left(m+\sum_{i=0}^{k}\left|T_{i}\right|\right)$ bound. To compute the argmax function efficiently we have to maintain a priority queue containing all unprocessed random vertices with a processed vertex along one of its outgoing edges. As we have $O(m)$ updates this costs only $O(m \log n)$ for one of the standard implementations of priority queues. Summing up this yields a $O\left(m \log n+\sum_{i=0}^{k}\left|T_{i}\right|\right)$ running time for Algorithm 1 and by considering also the time MEC for the MEC decomposition we obtain the desired bound.

Planning in Graphs. The algorithm for graphs works identically to the algorithm for MDPs but it does not need the priority queue. This is due to the fact that $Q$ is always nonempty and the MEC decomposition reduces to computing SCCs. We thus obtain a running time of $O\left(m+\sum_{i=1}^{n}\left|T_{i}\right|\right)$.

Theorem 6. Given a graph $G=(V, E)$, a starting vertex $s$ and a tuple of targets $\mathcal{T}=\left(T_{1}, \ldots, T_{k}\right)$, we can calculate 
whether there is a player-1 policy $\sigma_{1}$ at a start vertex $s$ for the objective $\operatorname{Seq}(\mathcal{T})$ in $O\left(m+\sum_{i=1}^{k}\left|T_{i}\right|\right)$ time.

Planning in Games. For game graphs with the tuple $\mathcal{T}=$ $\left(T_{1}, \ldots T_{k}\right)$ and starting vertex $s$, the basic algorithm performs $k$ player-1 attractor computations, starting with computing the attractor $S_{k}=\operatorname{Attr}_{1}\left(T_{k}\right)$ of $T_{k}$, then computing $S_{\ell}=\operatorname{Attr}_{1}\left(S_{\ell+1} \cap T_{\ell}\right)$ for $1 \leq \ell<k$, and finally returning $S_{1}$. This gives an $O(k \cdot m)$-time algorithm.

\section{Conditional Lower Bounds}

We present CLBs for game graphs based on the conjectures STC, SETH and OVC, which establish the CLBs for the third row of Table 1.

Sparse Game Graphs. For sparse game graphs, we present conditional lower bounds based on OVC. The reduction is an extension of Reduction 1, where we (a) again produce player-2 vertices instead of random vertices and (b) also every vertex of $S_{2}$ has an edge back to $s$. The reduction is illustrated in Figure 2. For the correctness recall that the game graph $\Gamma$ is constructed in such a way that there is no path between $x_{i}$ and $y_{j}$ iff they are orthogonal in the OV instance. Notice that each play starting at $s$ revisits $s$ every four steps and if there is no path between $x_{i}$ and $y_{j}$ then player 2 can disrupt player 1 from visiting a target $y_{j}$ by moving the token to $x_{i}$ whenever the token is in $s$. However, if there is no such $x_{i}$ and $y_{j}$, player 2 can not disrupt player 1 from $s$ because no matter which vertex $x_{i}$ player 2 chooses, player 1 has a policy to reach the next target set.

Theorem 7. There is no $O\left(m^{2-\epsilon}\right)$ or $O\left((k \cdot m)^{1-\epsilon}\right)$ algorithm (for any $\epsilon>0$ ) to check if a vertex $v$ has a winning policy for sequential reachability objectives in game graphs under Conjecture 4 (i.e., unless OVC and SETH fail).

Dense Game Graphs. For dense game graphs, we present a conditional lower bound based on BMM. The reduction extends Reduction 2, where we (a) produce player-2 vertices instead of random vertices and (b) every vertex in the fourth copy has an edge back to $s$. The reduction is illustrated in Figure 3. For the correctness of the reduction recall that there is a triangle in the graph $G$ iff there is a path from some vertex $v_{1 i}$ in the first copy of $G$ to the same vertex in the fourth copy of $G, v_{4 i}$. Player 2 then has a policy to always visit only $v_{1 i}$ from the first copy and only $v_{4 i}$ from the fourth copy which prevents player 1 from visiting target $T_{i}$.

Theorem 8. There is no combinatorial $O\left(n^{3-\epsilon}\right)$ or $O((k$. $\left.n^{2}\right)^{1-\epsilon}$ ) algorithm (for any $\epsilon>0$ ) to check if a vertex $v$ has a winning policy for sequential reachability objectives in game graphs under Conjecture 2 (i.e., unless STC and BMM fail). The bounds hold for dense game graphs with $m=\theta\left(n^{2}\right)$.

\section{Discussion and Conclusion}

In this work, we study several natural planning problems in graphs, MDPs, and game graphs, which are basic algorithmic problems in artificial intelligence. Our main contributions are a sub-quadratic algorithm for sequential target in MDPs, and quadratic conditional lower bounds. Note that graphs are a special case of both MDPs and game graphs, and the algorithmic problems are simplest for graphs, and in all cases, we have linear-time upper bounds. The key highlight of our results is an interesting separation of MDPs and game graphs: for basic target reachability, MDPs are harder than game graphs; for the coverage problem, both MDPs and game graphs are hard (quadratic CLBs); for sequential target reachability, game graphs are harder than MDPs.

Remark 5. Note that in Table 1 in the upper bounds for MDPs (second column) the term $m \cdot n^{2 / 3}$ appears consistently, which is the current best-known bound for the MECdecomposition problem. For all the upper bound results, any improvement for the MEC-decomposition bound also carries over and improves the $m \cdot n^{2 / 3}$ term in all entries of Table 1. Quite interestingly, for the coverage problem the CLB shows that the $k \cdot m$ term, which is present alongside the MEC-decomposition term, cannot be improved (this gives quadratic $C L B$ ), whereas for the sequential target problem, we present a sub-quadratic upper bound for MDPs.

Our lower bounds based on Conjecture 2 only apply to combinatorial algorithms (this excludes "algebraic methods" like fast matrix multiplication). However, notice that (a) our lower bounds based on Conjecture 4 are not restricted to combinatorial algorithms, (b) all existing algorithms for deciding almost-sure winning conditions in MDPs or winning conditions in games on graphs are indeed combinatorial and thus CLBs for combinatorial algorithms are of particular interest in our setting, and (c) Conjecture 2 also gives non-trivial lower bounds for non-combinatorial algorithms, i.e., $\tilde{\Omega}\left(\min \left\{n^{\omega}, m^{2 \omega /(\omega+1)}\right\}\right)$ lower bounds with $\omega$ being the matrix multiplication exponent.

In this work, we clarified the algorithmic landscape of basic planning problems with CLBs and better algorithms. An interesting direction of future work would be to consider CLBs for other polynomial-time problems in planning and $\mathrm{AI}$ in general. For MDPs with sequential targets, we establish sub-quadratic upper bounds, and hence the techniques of the paper that establish quadratic CLBs are not applicable. Other CLB techniques for this problem are an interesting topic to investigate as future work.

\section{Acknowledgments}

The authors are grateful to the anonymous referees for their valuable comments and suggestions to improve the presentation of the paper.

A. S. is fully supported by the Vienna Science and Technology Fund (WWTF) through project ICT15-003, the other authors are partially supported by that grant. K.C. is also supported by the Austrian Science Fund (FWF) NFN Grant No S11407-N23 (RiSE/SHiNE) and an ERC Starting grant (279307: Graph Games). For M.H the research leading to these results has received funding from the European Research Council under the European Union's Seventh Framework Programme (FP/2007-2013) / ERC Grant Agreement no. 340506 . 


\section{References}

Abboud, A., and Williams, V. V. 2014. Popular conjectures imply strong lower bounds for dynamic problems. In FOCS, 434-443.

Aghighi, M.; Bäckström, C.; Jonsson, P.; and Ståhlberg, S. 2016. Refining complexity analyses in planning by exploiting the exponential time hypothesis. Annals of Mathematics and Artificial Intelligence 78(2):157-175.

Bäckström, C., and Jonsson, P. 2017. Time and space bounds for planning. Journal of Artificial Intelligence Research 60:595-638.

Ballard, G.; Demmel, J.; Holtz, O.; and Schwartz, O. 2012. Graph expansion and communication costs of fast matrix multiplication. J. ACM 59(6):32:1-32:23.

Beeri, C. 1980. On the membership problem for functional and multivalued dependencies in relational databases. ACM Transactions on Database Systems (TODS) 5(3):241-259.

Bonet, B., and Geffner, H. 2000. Planning with incomplete information as heuristic search in belief space. In AIPS, 5261.

Bringmann, K., and Künnemann, M. 2015. Quadratic conditional lower bounds for string problems and dynamic time warping. In FOCS, 79-97.

Chatterjee, K., and Henzinger, M. 2014. Efficient and dynamic algorithms for alternating büchi games and maximal end-component decomposition. J. ACM 61(3):15:1-15:40.

Chatterjee, K.; Dvořák, W.; Henzinger, M.; and Loitzenbauer, V. 2016. Model and objective separation with conditional lower bounds: Disjunction is harder than conjunction. In LICS, 197-206.

Choset, H. M. 2005. Principles of robot motion: theory, algorithms, and implementation. MIT press.

Cimatti, A.; Pistore, M.; Roveri, M.; and Traverso, P. 2003. Weak, strong, and strong cyclic planning via symbolic model checking. Artificial Intelligence 147(1):35-84.

Clarke, Jr., E. M.; Grumberg, O.; and Peled, D. A. 1999. Model Checking. Cambridge, MA, USA: MIT Press.

Filar, J. A., and Vrieze, K. 1997. Competitive Markov Decision Processes. Springer.

Hansen, E. A., and Zilberstein, S. 1998. Heuristic search in cyclic and/or graphs. In AAAI, 412-418.

Henzinger, M.; Krinninger, S.; Nanongkai, D.; and Saranurak, T. 2015. Unifying and strengthening hardness for dynamic problems via the online matrix-vector multiplication conjecture. In Proceedings of the Forty-seventh Annual ACM Symposium on Theory of Computing, STOC '15, 2130. New York, NY, USA: ACM.

Hoffmann, J., and Brafman, R. 2005. Contingent planning via heuristic forward search with implicit belief states. In ICAPS, 71-88.

Howard, H. 1960. Dynamic Programming and Markov Processes. MIT Press.

Immerman, N. 1981. Number of quantifiers is better than number of tape cells. Journal of Computer and System Sciences 22(3):384-406.
Impagliazzo, R., and Paturi, R. 1999. Complexity of k-sat. In $C C C, 237-240$.

Impagliazzo, R.; Paturi, R.; and Zane, F. 1998. Which problems have strongly exponential complexity? In FOCS, 653662.

Kaelbling, L. P.; Littman, M. L.; and Cassandra, A. R. 1998. Planning and acting in partially observable stochastic domains. Artificial Intelligence 101(1):99-134.

Kress-Gazit, H.; Fainekos, G. E.; and Pappas, G. J. 2009. Temporal-logic-based reactive mission and motion planning. IEEE Transactions on Robotics 25(6):1370-1381.

Kronegger, M.; Pfandler, A.; and Pichler, R. 2013. Parameterized complexity of optimal planning: A detailed map. In IJCAI, 954-961.

LaValle, S. M. 2006. Planning algorithms. Cambridge University Press.

Le Gall, F. 2014. Powers of tensors and fast matrix multiplication. In ISSAC, 296-303.

Mahanti, A., and Bagchi, A. 1985. AND/OR graph heuristic search methods. J. ACM 32(1):28-51.

Maliah, S.; Brafman, R.; Karpas, E.; and Shani, G. 2014. Partially observable online contingent planning using landmark heuristics. In ICAPS, 163-171.

Palacios, H., and Geffner, H. 2007. From conformant into classical planning: Efficient translations that may be complete too. In ICAPS, 264-271.

Papadimitriou, C. H., and Tsitsiklis, J. N. 1987. The complexity of Markov decision processes. Mathematics of $\mathrm{Op}$ erations Research 12:441-450.

Puterman, M. L. 1994. Markov Decision Processes. John Wiley and Sons.

Russell, S. J., and Norvig, P. 2010. Artificial Intelligence A Modern Approach (3rd ed.). Pearson Education.

Tarjan, R. 1972. Depth-first search and linear graph algorithms. SIAM Journal on Computing 1(2):146-160.

Vassilevska-Williams, V. 2018. On some fine-grained questions in algorithms and complexity. In ICM, to appear.

Williams, V. V., and Williams, R. 2018. Subcubic equivalences between path, matrix and triangle problems. J. ACM. to appear. preliminary version available at http://people. csail.mit.edu/virgi/tria-mmult-jv.pdf.

Williams, R. 2005. A new algorithm for optimal 2-constraint satisfaction and its implications. Theoretical Computer Science 348(2):357 - 365.

Williams, V. V. 2012. Multiplying matrices faster than coppersmith-winograd. In STOC, 887-898. 